\title{
Age-related cerebral small vessel disease and inflammaging
}

Tiemei $\mathrm{Li}^{1,2}$, Yinong Huang ${ }^{1,3}$, Wei Cai ${ }^{1,2}$, Xiaodong Chen ${ }^{1,2}$, Xuejiao Men ${ }^{1,2}$, Tingting Lu ${ }^{1,2}$, Aiming Wu ${ }^{1,2}$ and Zhengqi Lu $\mathbb{B}^{1,2}$

\begin{abstract}
The continued increase in global life expectancy predicts a rising prevalence of age-related cerebral small vessel diseases (CSVD), which requires a better understanding of the underlying molecular mechanisms. In recent years, the concept of "inflammaging" has attracted increasing attention. It refers to the chronic sterile low-grade inflammation in elderly organisms and is involved in the development of a variety of age-related chronic diseases. Inflammaging is a long-term result of chronic physiological stimulation of the immune system, and various cellular and molecular mechanisms (e.g., cellular senescence, immunosenescence, mitochondrial dysfunction, defective autophagy, metaflammation, gut microbiota dysbiosis) are involved. With the deepening understanding of the etiological basis of age-related CSVD, inflammaging is considered to play an important role in its occurrence and development. One of the most critical pathophysiological mechanisms of CSVD is endothelium dysfunction and subsequent blood-brain barrier (BBB) leakage, which gives a clue in the identification of the disease by detecting circulating biological markers of BBB disruption. The regional analysis showed blood markers of vascular inflammation are often associated with deep perforating arteriopathy (DPA), while blood markers of systemic inflammation appear to be associated with cerebral amyloid angiopathy (CAA). Here, we discuss recent findings in the pathophysiology of inflammaging and their effects on the development of age-related CSVD. Furthermore, we speculate the inflammaging as a potential target for future therapeutic interventions to delay or prevent the progression of the age-related CSVD.
\end{abstract}

\section{Facts}

- The latest cellular and molecular mechanisms of inflammaging include cellular senescence, immunosenescence, mitochondrial dysfunction, defective autophagy, metaflammation and gut microbiota dysbiosis.

- The relationship between inflammaging and age-
Correspondence: Zhengqi Lu (Izq1828@outlook.com)

'Department of Neurology, the Third Affiliated Hospital of Sun Yat-sen University, Guangzhou, Guangdong 510630, China

${ }^{2}$ Mental and Neurological Diseases Research Center, the Third Affiliated Hospital of Sun Yat-sen University, Guangzhou, Guangdong 510630, China

Full list of author information is available at the end of the article

These authors contributed equally: Tiemei Li, Yinong Huang

Edited by F. Pentimalli related CSVD is explored from the two following aspects: vascular inflammation and DPA; systemic inflammation and CAA.

- The contributions of inflammaging in age-related CSVD are potential target for future therapeutic interventions.

\section{Open questions}

- Does inflammaging play a role in explaining the pathophysiological interactions between DPA and CAA?

- Future research should focus on the co-relationship between gut microbiota and inflammaging in agerelated CSVD patients.

- Clinical studies are needed to assess the effectiveness 
of inflammaging-targeted interventions for agerelated CSVD.

\section{Introduction}

Cerebral small vessel disease (CSVD) is defined as a group of CSVD, in which cerebral small arteries, arterioles, capillaries, venules, small veins are attributed to atherosclerotic, genetic, idiopathic, infectious, immunemediated or other secondary etiologies ${ }^{1}$. The age-related CSVD includes deep perforating arteriopathy (DPA) and $\beta$-amyloid $(\mathrm{A} \beta)$ related cerebral amyloid angiopathy (CAA). Age-related CSVD is most prevalent among the elderly and contributes to high global disease burden of stroke and vascular dementia. Advanced age and hypertension are the most accepted epidemiologic risk factors ${ }^{2}$. An increased incidence of age-related CSVD accompanies the steady worldwide increase in life expectancy, affects about $5 \%$ of people aged 50 years and almost everyone older than 90 years, leading to a huge social and economic burden ${ }^{1}$.

Aging is characterized by a progressive decline in multiple physiological processes, which makes the elderly a highly vulnerable population. Aging is a major risk factor for chronic non-communicable diseases (e.g. diabetes, cancer, cardiovascular diseases, CSVD), among which inflammaging plays an important role $^{3}$. The term inflammaging describes the condition of chronic sterile low-grade inflammation observed in older organisms ${ }^{4}$. Inflammaging is the long-term result of chronic physiological stimulation of the immune system, and possess various cellular and molecular mechanisms, including cellular senescence, immunosenescence, mitochondrial dysfunction, defective autophagy, metaflammation, and gut microbiota dysbiosis ${ }^{5}$ (Fig. 1).

An important pathophysiological mechanism of CSVD is endothelium dysfunction and subsequent blood-brain barrier (BBB) leakage, which gives a clue in the identification of the disease through circulating biological mar$\mathrm{kers}^{2}$. As deepening understanding in the etiological basis of age-related CSVD, inflammaging has been proposed as a candidate factor. Typical markers of inflammation can be classified as systemic inflammatory factors (e.g., Creactive protein, interleukin-6) or vascular inflammation/ endothelial dysfunction (e.g., homocysteine, von Willebrand factor). A previous study indicated a strong correlation between vascular inflammatory markers and CSVD, especially in CSVD patients with stroke ${ }^{6}$. Meanwhile, longitudinal survey illustrated that baseline expression levels of systemic inflammatory factors could predict the severity of the subsequent CSVD. More importantly, the regional analysis showed that systemic and vascular inflammation was associated with two different subtypes of CSVD (CAA and DPA), respectively. To be specific, markers of vascular inflammation tended

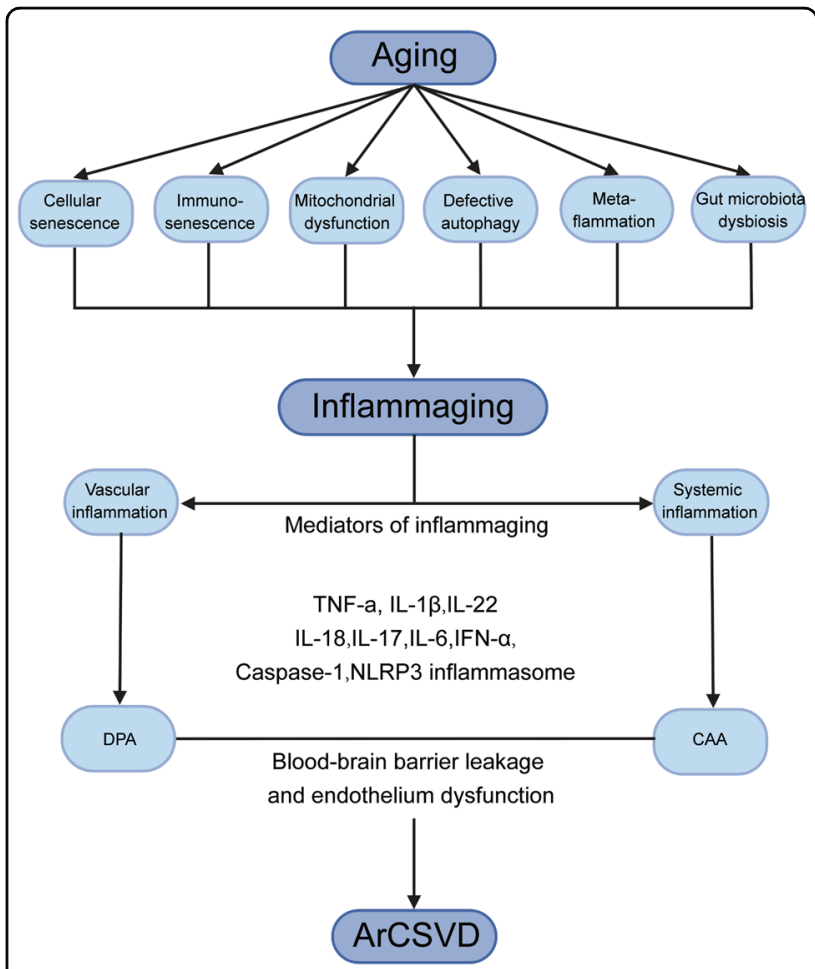

Fig. 1 The deep interactions between aging, inflammaging, and age-related CSVD. As aging, several cellular and molecular mechanisms lead to chronic inappropriate activation of the immune system. This complex interaction between genetic susceptibility and risk stimuli (both exogenous and endogenous) contributes to the continuous activation of a limited range of confounding sensors which triggers inflammaging (upper part of the box). The resulting synthesis and release of different inflammatory mediators are related to the common pathophysiological mechanisms of age-related diseases. For age-related CSVD, regional analyses showed that blood markers of vascular inflammation were associated with deep perforating arteriopathy (DPA), while blood markers of systemic inflammation were associated with cerebral amyloid angiopathy (CAA), both of which were closely related to the critical pathophysiological mechanisms of blood-brain barrier leakage and endothelial dysfunction (lower part of the box).

to be associated with DPA (e.g., basal ganglia), whereas systemic inflammation seemed to be associated with CAA (e.g. centrum semiovale) ${ }^{6}$ (Fig. 1).

This review focuses on the latest research of inflammaging, systemic inflammatory biomarkers and pathophysiological progression of CSVD, and explores the potential link between inflammaging and age-related CSVD. Besides, we discuss the potential role of inflammaging as a target for future therapeutic interventions to delay or prevent the progression of the age-related CSVD.

\section{Inflammaging}

The term inflammaging was coined in the early 21 st century to refer to a persistent sterile, low-grade systemic pro-inflammatory status that occurs during aging process 
in mammals ${ }^{4}$. Inflammation is a valid defense mechanism to resist harmful substances invading the body and maintain homeostasis. However, the harmful effects of chronic subclinical inflammation on the body might be an essential risk factor for increasing the incidence of degenerative diseases (e.g., osteoporosis) and metabolic diseases (e.g., type 2 diabetes) in the elderly ${ }^{7}$.

The innate and adaptive immune systems protect our bodies from harmful substances and inappropriate stimuli, which promote the development of inflammaging. These stimuli include pathogens such as bacteria, viruses, fungi and parasites (non-self), endogenous cell debris and misplaced molecules (self), and nutrients and gut microbiota (quasi-self) ${ }^{8}$. A limited number of sensors in the body sense the stimuli, whereas their degeneracy allows them to identify many signals and activate the downstream cascades. Pattern recognition receptors (PRRs), including toll-like receptors (TLRs) ${ }^{9}$, NOD-like receptors, cyclic GMP-AMP synthetase (cGAS), aryl hydrocarbon receptor (AHR), have the degeneracy of biological functions. These receptors can recognize different stimuli produced by non-self molecules including viral and bacterial products (pathogen-associated molecular patterns, PAMPs), self molecules (termed damage-associated molecular patterns, DAMPs) and nutritional and metabolic products from the gut microbiota (which could be considered as quasi-self $)^{8}$. Inflammaging is an unpredicted consequence of the evolution-driven degeneracy of damage sensors ${ }^{10}$ (Fig. 2).

Inflammaging is a situation under which immunity exhibits antagonistic pleiotropy during aging. It involves a variety of different cellular and molecular mechanisms,

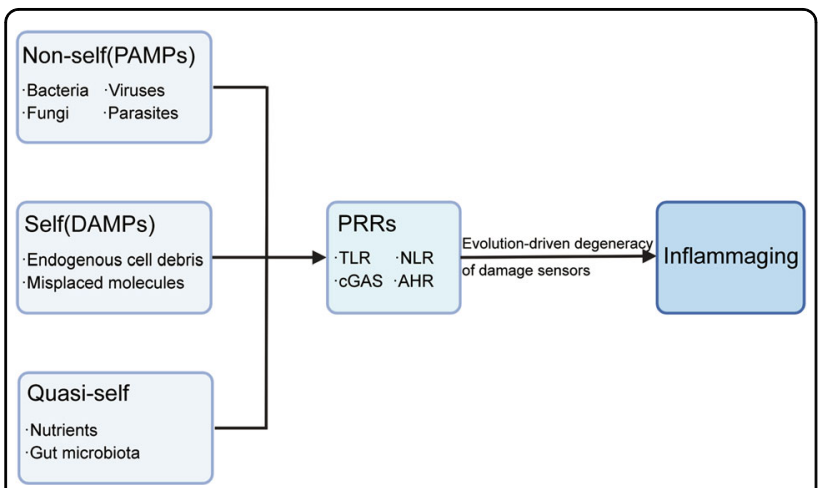

Fig. 2 The degeneracy of the damage sensor triggers inflammaging. Exogenous and endogenous danger stimuli interact with pattern recognition receptors (PRRs) expressed on the cell surface and in the cytoplasm. Danger molecules can be non-self (pathogen-associated molecular patterns, PAMPs), self (damageassociated molecular patterns, DAMPs) and quasi-self (nutritional and metabolic products from the gut microbiota). These multitude of stimuli converge on the same evolutionarily selected promiscuous sensors, and trigger inflammaging. which are synergistic and mutually promoting ${ }^{11}$ as detailed below.

\section{Cellular senescence}

The leading self-stimuli of inflammaging are the accumulation of damaged macromolecules and cellular debris, which could chronically inhibit the capacity of multiple tissues to surveil and repair damage ${ }^{12}$. Thus, cellular senescence may be the cause of inflammaging. Growing evidence shows that senescent cells (SCs) exert detrimental effects on the tissue micro-environment by generating pathological facilitators. Accordingly, it has been suggested that SCs contribute to the development of inflammaging through the generation of senescence-associated secretory phenotype (SASP), which consists of a variety of soluble factors, such as proinflammatory mediators (e.g., IL-6, IL-8) and matrixdegrading molecules ${ }^{13}$. Non-degradable wastes in the body chronically activates innate immunity via self-stimuli of DAMPs, in which macrophages play a central role ${ }^{14}$. Innate immune cells subsequently activate classical pro-inflammatory pathways such as nuclear factor kappa-light-chain enhancer of activated B cells (NF-kB) and signal transducer and activator of transcription (STAT), and secrete a large amount of proinflammatory cytokines/chemokines (e.g., IL-6) ${ }^{15,16}$.

In addition to immune cells and tissue cells, tissueresident stem cells, especially mesenchymal stromal cells (MSCs), are also affected by cellular senescence ${ }^{17}$. Similar to other adult stem cells, MSCs possess a limited capacity of self-renewal. The aging process leads to the ageassociated decline in cell number and dysfunction of $\mathrm{MSCs}^{18}$. Aged MSCs existing in vivo may participate in the development of inflammaging exogenously through the following mechanisms: a. Aged MSCs produce large amounts of cellular wastes in vivo, which act as an important source for DAMPs and exacerbate the onset and development of inflammaging. b. Aged MSCs acquire SASP by producing a robust amount of pro-inflammatory cytokines, including IL-6, IL-8, interferon-gamma (IFN$\gamma)$, monocyte chemoattractant protein (MCP)-1, and matrix metalloproteinases (MMP2, TIMP2). c. MSCs possess potent immunomodulatory capacity (such as promoting macrophage polarization from M1 to M2), whereas the aging process compromised these beneficial effects. d. MSC is one of the critical components of the hematopoietic stem cell (HSC) niche, which plays a crucial role in maintaining HSC homeostasis and bone marrow microenvironment. Age-associated mutations hinder niche functions of MSCs and compromise their hematopoietic supportive function in the elderly ${ }^{19}$.

\section{Immunosenescence}

In addition to inflammaging (over-reaction), an important feature of the immune system aging is immunosenescence (insufficiency) ${ }^{20}$. Immunosenescence refers to 
the destruction of immune organ structure and the dysfunction of the immune response, which leads to a decline in the preservation and enhancement of many immune functions. It is the result of combined actions of innate immunity and adaptive immunity ${ }^{21}$. Although immunosenescence and inflammaging seem to be antipathic phenotypes, there are many overlaps in their mechanisms and progression from the perspective of immune dysfunction ${ }^{20}$.

In the immune system, there is an innate compartment, consisting of neutrophils monocytes/macrophages, natural killer cells, and dendritic cells, and an adaptive compartment, composed of $\mathrm{B}$ and $\mathrm{T}$ cells ${ }^{22}$. As the first barrier against pathogens, the immunosenescence of the innate immune system is extremely complex and seems to reflect dysregulation, not just dysfunction. In fact, some responses of the innate immune system decrease with age, but the age-related hyperreactivity of innate immunity has also been reported ${ }^{23}$. Immunosenescence of the adaptive immune system is characterized by reduced naive $\mathrm{CD} 4^{+}$ and $\mathrm{CD}^{+} \mathrm{T}$ cell counts, an imbalance of $\mathrm{T}$ cell subsets, and a decrease in $\mathrm{T}$ cell receptor (TCR) repertoire and signaling. The development and selection of $\mathrm{T}$ cells occur in the thymus. Thymus degeneration leads to a decrease in the frequency and number of naive $T$ cells, an increase in the number of terminally differentiated $\mathrm{T}$ cells, and a decrease in the expression of $\mathrm{TCR}^{24}$. The numerical reduction in naïve $\mathrm{T}$ cells and TCR-reduced repertoire result in T cells dysfucntion ${ }^{25}$. Similarly, there is a decline in B cells and a reduction in antibody production ${ }^{26}$. B cells follow a well-defined developmental process, starting from naïve cells that do not produce specific antibodies isotype, to the establishment of peripheral B cell pools that maintains maturity through self-renewal ${ }^{27}$. The most significant alterations of $\mathrm{B}$ cells during aging are loss of naïve $B$ cells, impaired capacity to response to new antigens, reduction of clonal expansion of memory cells, and weakened antibodies function ${ }^{28}$.

\section{Mitochondrial dysfunction and defective autophagy}

Mitochondrial metabolism and autophagy are the two most metabolically active cellular processes and play an important role in regulating the lifespan of organisms ${ }^{29}$. Mitochondria control a large number of cellular processes $^{30}$, not only by controlling the production of ATP, but also by acting as biosynthetic and signaling centers. Autophagy is a cellular process responsible for degrading damaged organelles and protein aggregates. Autophagy degrades several organelles, one of which deserves particular attention is autophagy-mediated mitochondrial degradation called mitophagy ${ }^{31}$.

There is an apparent interconnection between mitochondria and autophagy, both of which promotes the process of inflammaging ${ }^{32}$. The crosstalk of mitochondrial dysfunction and defective autophagy favors the activation of several inflammatory pathways. For example, autophagy defects promote the accumulation of dysfunctional mitochondria, leading to the direct release of large amounts of mitochondrial DNA (mtDNA) into the cytoplasm. MtDNA in the cytoplasm induces the activation of caspase- 1 and subsequent production of $\mathrm{IL}-1 \beta^{33,34}$. Besides, mitochondria are the signaling hubs ${ }^{33}$ that regulate intracellular calcium pool and reactive oxygen species (ROS) levels, both of which are classical inflammatory mediators ${ }^{35,36}$. Maintenance of mitochondrial and endoplasmic reticulum contact sites regulates leukocyte migration and lymphocyte activation by balancing intracellular calcium pool and regulating autophagy induc$\operatorname{tion}^{37}$. In the oxidation-inflammation theory of aging, it is proposed that accumulated mtDNA mutations could disrupt mitochondrial respiratory chain and leads to excessive mitochondrial ROS (mtROS) production. In turn, this accelerates the emergence of new mutations in mtDNA (leading to cellular senescence) and aggravates the inflammaging process. In addition, aging leads to the further production of ROS and inflammatory mediators, namely oxi-inflamm-aging, forming a vicious cycle ${ }^{38}$.

\section{Metaflammation and gut microbiota dysbiosis}

Metaflammation refers to metabolic inflammation caused by nutrient excess or overnutrition ${ }^{8}$, which exists in chronic age-related metabolic diseases (e.g. type 2 diabetes, obesity). Metaflammation is the most convincing mechanism linking nutritional disorders to inflammaging, indicating that the inflammatory milieu of metabolic cells, tissues and organs is altered due to the high nutrient intake $^{8}$. A pro-inflammatory milieu characterized by highcaloric intake promotes insulin resistance ${ }^{39,40}$. The level of pro-inflammatory cytokines increases during the process of inflammaging, which promotes the infiltration of immune cells in insulin-responsive tissues (e.g., fat and muscle), thereby increase local oxidative stress and inflammatory response, and decrease the expression of insulin receptors ${ }^{41,42}$. Besides, in the post-prandial state, residual chylomicrons and very low-density lipoprotein (VLDL) bind to endothelial cells (ECs) and circulating leukocytes, resulting in acute cell activation and a surge of adhesion molecules, cytokines, and oxidative stress, which ultimately leads to inflammaging ${ }^{43-45}$.

As a cross between metabolism and inflammation, the gut microbiota has a central role in both metaflammation and inflammaging regulation ${ }^{43}$. The human gut microbiota is a highly diverse ecosystem, composed of trillions of bacteria, each part of which has a specific role and respond to a variety of signals from the host. These microbiota alter their own activities to achieve mutualism with the host ${ }^{46}$. Beneficial and potentially pathogenic microorganisms have similar epitopes, gain immunological features through their evolution, and 
acquire immune tolerance in the host ${ }^{47-49}$. An analysis of the human gut microbiota showed that among Italian individuals (aged 22-109 years), the core population of gut microbiota exhibited a decrease in diversity and relative abundance with aging $^{50}$. In the gut microbiota of the elderly, there is an enrichment of proteobacteria and a decrease in butyrateproducing bacteria, which leads to the decrease of Treg cells, indirect increase of Th17 cells and the generation of proinflammatory cytokines including IL-6, IL-8, IL- $17^{51}$. In mice studies, the gut microbiota of aged mice was inoculated into young germ-free mice, causing an inflammatory response with an increased proportion of $\mathrm{T}$ helper (Th) cell subsets and increased levels of inflammatory markers such as tumor necrosis factor (TNF). This might be related to the increased inflammatory potential of the gut microbiota of aged mice ${ }^{52,53}$.

Aging and age-related diseases share these basic mechanism pillars, which ultimately converge on inflammation to a large extent. In the aging process, inflammaging promotes the occurrence of age-related diseases. Next, we will further discuss the relationship between inflammaging and age-related CSVD.

\section{The relationship between inflammaging and age- related CSVD}

Inflammaging is increasingly recognized as a risk factor for dementia, stroke, and CSVD ${ }^{6}$. Inflammaging not only works through the aging immune system, but also interacts with traditional cerebrovascular risk factors (e.g. obesity, hypertension, and type 2 diabetes) to exacerbate their harmful effects. In CSVD patients, lesions such as repeated mild stroke lead to BBB leakage, central nervous system antigen release into the peripheral circulation, and lymphocyte infiltration into brain tissue and related cerebral dysfunction ${ }^{54}$. In turn, brain dysfunction may further damage the immune system, forming a vicious circle $^{55}$. Thus, the relationship between inflammaging and the development of age-related CSVD deserves our attention.

Circulating biomarkers of CSVD inflammation were classified as markers of systemic inflammation and markers of vascular inflammation/endothelial dysfunction ${ }^{6}$. Besides, four core MRI features have been identified as imaging markers of CSVD, namely white matter hyperintensities (WMH), lacunae, cerebral microhemorrhage (CMB), and perivascular space enlargement (EPVS $)^{56}$.

We will explore the relationship between inflammaging and age-related CSVD from the following two aspects: vascular inflammation and DPA; systemic inflammation and CAA.

\section{Vascular inflammation and deep perforator arteriopathy}

DPA mainly affects small arteries, veins, arterioles, venules and capillaries (diameter ranging between 100 and 400 microm). These small arteries are derived from deep perforating vessels of large vessels at the base of the brain or penetrating cortical/medullary vessels from superficial medium-sized arteries ${ }^{57}$. The main lesions in the blood supply area of DPA result in WMH in the periventricular region, deep CMB and EPVS in the basal ganglia, etc. For anatomical reasons, the supply vessels in these areas are more likely to narrow due to atherosclerosis, especially in elderly patients with hypertension and diabetes ${ }^{58-60}$. Furthermore, BBB and endothelial dysfunction seem to play important roles in DPA. Jandke et al. $^{61}$ proved that rats with DPA exhibited injured endothelial tight junctions and leakage of proteins deposited surrounding the small vessel walls.

Pro-inflammatory cytokines and inflammatory cells are involved in the formation of atherosclerosis during inflammaging ${ }^{62,63}$. Pro-inflammatory cytokines impair ECs and BBB function, and induce the expression of adhesion molecules and chemokines recruiting leucocytes to cerebral lesions. In the spontaneously hypertensive rat (SHR), peripheral blood and infiltrating immune cells (e.g., T cells, NK cells) were found to expand in the cerebral blood vessels, leading to inflammation, endothelial dysfunction, and ischemia in this area ${ }^{64}$. The increased expression of adhesion molecules might lead to an increased number of $\mathrm{T}$ cells in SHR brains. On one hand, $\mathrm{T}$ cells adhering to the lumen side of brain microvessels might be part of the systemic adaptive immune response to angiogenesis antigens during hypertension. On the other hand, $\mathrm{T}$ cells directly promoted endothelial dysfunction, and might impair brain perfusion by microvascular plugging and thrombosis ${ }^{65}$. In addition, a variety of anti-endothelial cell antibodies were found in the serum of CSVD patients, suggesting a possible mechanism that B cell activation promotes endothelial dysfunction ${ }^{66}$. During inflammaging, monocytes activate pathways that promote inflammatory polarization, driving ROS production and hypertension ${ }^{67}$. Macrophages infiltrating vascular walls produce elevated levels of ROS, reduce the use of $\mathrm{NO}$, promote the expression of adhesion molecules, stimulate vascular smooth muscle cell (VSMC) hypertrophy, and activate matrix metalloproteinases, all of which are involved in vascular remodeling and dysfunction ${ }^{68}$.

Inflammasomes are multiprotein signaling complexes. They play a key role in the mediation of innate inflammatory responses and are assembled in response to a wide range of stimuli including both PAMPs and DAMPs ${ }^{69}$. Although several types of inflammasomes have been identified so far, the best characterized is the NOD-like receptor family pyrin domain containing 3 (NLRP3) inflammasome ${ }^{70}$. The activation of NLRP3 inflammasome requires two steps to strictly regulate its functions. First, the initiation step increases the expression of individual inflammasome components and assembles them into macromolecular multimers. Then, various potentially 
harmful stimuli provide a second signal that endows the inflammasome component caspase- 1 with activity. This proteinase cleaves the inactive precursors of the proinflammatory cytokines such as IL- $1 \beta$ and IL-18, conferring them biological activity ${ }^{71}$. During inflammaging, the continuous increase of inflammatory mediators and the decrease of anti-inflammatory cytokines (e.g., IL-10, adiponectin) may exacerbate vascular extracellular matrix remodeling and arterial stiffening, thereby accelerating plaque formation ${ }^{72}$.

NLRP3 inflammasome is recognized as an important mediator linking atherosclerosis and inflammaging ${ }^{73}$. Atherosclerotic lesions produce DAMPs (e.g., cholesterol crystals) that activate NLRP3 inflammasomes in macrophages and generate active forms of pro-inflammatory cytokines (such as IL-1 $\beta$ and IL-18) ${ }^{67}$. The resulting inflammatory environment leads to the formation of complex plaques by mediating cellular recruitment and the generation of foam cells and fatty streaks. Besides, IL$1 \beta$ and IL-18,as the main products of NLRP3 activation, trigger the expression of IL- 6 in many types of cells ${ }^{74}$. This cascade reaction greatly amplifies the inflammasome signal. IL-6 can promote thrombotic events by producing of fibrinogen and plasminogen activator inhibitor-1 in the liver, thereby making the blood more coagulable and impairing fibrinolysis ${ }^{75}$.

Accumulated SCs are detected in advanced atherosclerotic plaques by measuring specific markers such as senescence-related $\beta$ galactosidase $(S A \beta G), p 16$, and tumor suppressor replacement reading frame (ARF). Compared with age-matched healthy vessels, the proportion of SABG-positive cells in the intimal and medial layers of atherosclerosis increase ${ }^{76,77}$. SCs contribute to the development of inflammaging through $\mathrm{SASP}^{78}$. Wang et al. ${ }^{79}$ found that the increase of matrix metalloproteinases enhances the decomposition of the extracellular matrix and promotes the reconstruction of advanced atherosclerotic plaques. The decomposition of the extracellular matrix promotes the migration of VSMC from media, mediates the compensatory enlargement of arteries, and weakens the protective fibrous cap of plaques ${ }^{80}$.

Metaflammation and gut microbiota dysbiosis play an important role in arterial hypertension and vascular dysfunction induced by inflammaging. Overweight and obese patients tend to suffer from a state of metaflammation. In the post-prandial period, their circulating VLDL could induce the expression of adhesion molecules, cytokines, and pro-oxidants in ECs and leucocytes, which is conducive to the occurrence of vascular inflammation ${ }^{81,82}$. Moreover, insulin resistance mediated by metabolic inflammation is a co-recognized vascular risk factor associated with aging. In this way, inflammaging amplifies the vicious cycle of obesity, insulin resistance, aging, and vascular dysfunction ${ }^{83}$. Besides, aging increases the accumulation of monocytes and lymphocytes in adipose tissue $^{84}$, which are considered as critical mediators of inflammation and oxidative stress in arterial hypertension.

Research on cardiovascular (CV) disease have reported the presence of bacteria in atherosclerotic plaques ${ }^{85,86}$, suggesting that gut microbiota may influence age-related $\mathrm{CV}$ inflammation. In our recent study, we found that the altered microflora also affected the pathophysiology of atherosclerotic CSVD (aCSVD). Besides, we evaluated the inflammatory status of aCSVD patients by quantifying the mRNA levels of inflammaging markers in circulating leukocytes. The expression levels of the inflammaging markers (including IL-17A, TNF- $\alpha$, IL-6, and IFN $\alpha$ ) increased in aCSVD patients compared with the healthy control. The tested inflammaging markers displayed prognostic significance, expression of IL-1 $\beta$, IL-6, IFN- $\alpha$, and IL-17A increased the predicting sensitivity of detrimental imageology signs (unpublished data).

\section{Systemic inflammation and CAA}

Sporadic (non-inflammatory) CAA is specifically characterized by the progressive deposition of amyloid $\beta 40$ (A 340 ) protein (to a lesser extent, $A \beta 42$ ): (i) capillary CAA (CAA-type 1), which mainly affect the walls of capillaries and the surrounding neuropilcapillary CAA (CAA-type 1), (ii) arterioles and rarely veins (CAA-type 2), which mainly involve noncapillary blood vessels such as small to medium-sized arteries of parenchymal and leptomeningeal ${ }^{87}$. Regional studies shown that systemic inflammation was preferentially associated with vascular injury in areas commonly associated with CAA pathology (lobular regions supplied by cortical and leptomeningeal vessels $)^{6}$.

$A \beta$ is derived from the enzymatic cleavage of transmembrane amyloid precursor proteins in cells (e.g., neurons and oligodendrocytes). Soluble $A \beta$ can be removed from the brain by various clearance systems, including enzymatic degradation and cellular uptake, transport across the BBB, interstitial fluid (ISF) bulk flow, and cerebrospinal fluid (CSF) absorption into the circulatory and lymphatic systems ${ }^{88,89}$. In addition, mechanisms eliminating $A \beta$ out of the brain depend on the integrity of cerebral microvessels, whereas the risk of vascular wall damage increases with age.

As sentinels of the brain, microglia exhibit multiple responses to external stimuli and play an important role in $A \beta$ clearance. During aging, microglia acquire an activated phenotype, morphologically characterized by reduced branches and increased cell soma volume; functionally defined by the release of pro-inflammatory cytokines such as IL- $1 \beta$, TNF- $\alpha$, and IL- $6^{90-92}$. The inflammatory responses initiated by microglia involves multiple inflammasomes, among which NLRP3 inflammasome is the most characteristic and most widely 
implicated regulators of IL- $1 \beta$ and IL- $18^{93,94}$. In a murine model assessing microglial dynamics in the context of systemic inflammation, 3-dimensional and quantitative analyses of microglia revealed that systemic inflammation (i) briefly affected microglia in an age-dependent manner; (ii) increased $A \beta$ deposition by affecting microglial cell clearance; (iii) increased microglial cell proliferation and served as a marker of disease acceleration. Moreover, the activation of NLRP3 inflammasome could be identified as a vital mediator of these effects ${ }^{95}$.

Chen Y et al. ${ }^{96}$ found that NLRP3 activation could impair endothelial permeability and barrier function under stress conditions such as hyperlipidemia. In highfat diet-fed mice, the harmful adipose factor visfatin increased in the serum, which acted on the ECs, activated NLRP3 to promote the release of downstream high mobility group box-1 protein 1 (HMGB1), and further caused a downregulation of interendothelial connexin ZO-1, ZO-2, occludin, and VE-cadherin, leading to increased endothelial permeability and impaired barrier function. Moreover, elevated oxidative stress and defective autophagy also activate NLRP3 inflammasome in senescent endothelium ${ }^{97}$. This process further aggravates vascular endothelial dysfunction, compromises $A \beta$ clearance and promotes the occurrence and development of CAA.

In addition to innate immune cells, the response of adaptive immune cells, especially $\mathrm{T}$ cells, to brain amyloidosis has also received increasing attention. In transgenic ( $\mathrm{tg}$ ) mouse models of AD-like cerebral amyloidosis, studies shown that brain amyloidosis promoted $\mathrm{T}$ cell infiltration but interfered with local antigen presentation and $\mathrm{T}$ cell activation. In the long term, the ability of microglia and macrophages to remove $A \beta$ might be reduced in the absence of $\mathrm{T}$ cell-derived stimulants and/ or in response to $\mathrm{T}$ cell-derived inhibitory signals ${ }^{98}$.

The brain-gut axis refers to the bidirectional, continuous communication between the central nervous system and the gastrointestinal tract ${ }^{99}$. The term has recently been extended to the brain-gut-microbiota axis. There is increasing evidence that gut microbiota affects brain-gut interactions at different time points (from early life to aging) and at different levels (from the lumen to the central nervous system). Gut microbiota provides twoway communication through immune, hormone, and neuronal signals ${ }^{100}$. Inflammaging causes alterations in gut microbiota composition and decreases in microbiota diversity and stability, resulting in the destruction of the intestinal barrier. Disrupted intestinal barrier further increase circulating pro-inflammatory cytokines and bacterial derivatives, triggering systemic inflammation and BBB damage ${ }^{101}$.

Gut microbiota may affect the function of the central nervous system by directly synthesizing various neurotransmitters and neuromodulators. For example, signals from the gut microbiome may regulate the function of intestinal enterochromaffin cells, which produce different hormones and neurotransmitters, such as serotonin $^{102}$. Moreover, the results of studies in germ-free mice confirmed that microbiota may be mediated by bacterial metabolites short-chain fatty acids (SCFAs), thereby affect microglia maturation and reduce $\mathrm{BBB}$ permeability $^{100}$.

The diverse gut microbiota produce large amounts of lipopolysaccharide (LPS), amyloid proteins, and various microbial exudates ${ }^{103-105}$. Microbial and cerebral amyloids are biologically similar in higher-order structure, PAMPs composition, and physic-chemical characteristics, despite of their difference from the human A $\beta 1-42$ amino acid sequences ${ }^{104}$. In addition, they are also recognized by the same TLR2 / TLR1 receptor system as A $\beta 42$, which strongly activates the production of pro-inflammatory cytokines, particularly IL-17 and IL-22. In the innate immune system, LPS activates TLRs expressed in microglia cells, which recognize common injuries or PAMPs ${ }^{106}$. LPS activates the TLR4 receptor and promotes inflammation by interacting with CD14 and MD-2 proteins. The activation of TLR4 in CD14 also mediates the inflammatory response to $A \beta^{107}$. Secretion from the total microbiome constitute a very powerful class of proinflammatory complement and innate immune activators. They possess great potential for inducing proinflammatory cytokines, complement activation and altering immunogenicity in the brain. Both amyloid proteins and LPS are potent activators of the receptors for advanced glycosylation end products (RAGE) and TLRs. This pathogenic effect enhances amyloid accumulation and increases the inflammatory response ${ }^{108}$. In daily life, people are also exposed to large amounts of LPS and amyloid proteins continuously produced by the gut microbiota. Such exposure may be harmful to health. This deleterious effect is more evident when the gastrointestinal mucosa and $\mathrm{BBB}$ are remodeled during inflammaging (Fig. 3) ${ }^{104,105}$. However, current studies on gut microbiota of neuropathic diseases with the amyloidogenic component are mostly focused on Alzheimer's disease, with no animal or clinical research on CAA.

DPA and CAA are extreme outcomes of the pathologic continuum of age-related CSVD. Endothelial injury, destruction of the $\mathrm{BBB}$ and impaired perivascular $\mathrm{A} \beta$ drainage are the main mechanisms of age-related CSVD ${ }^{2}$. A comprehensive and in-depth understanding of the relationship between inflammaging and age-related CSVD will help us propose targeted anti-CSVD treatment plans.

\section{Possible therapeutic interventions}

Treatment of age-related CSVD includes specific and non-specific treatment. Non-specific treatment includes 


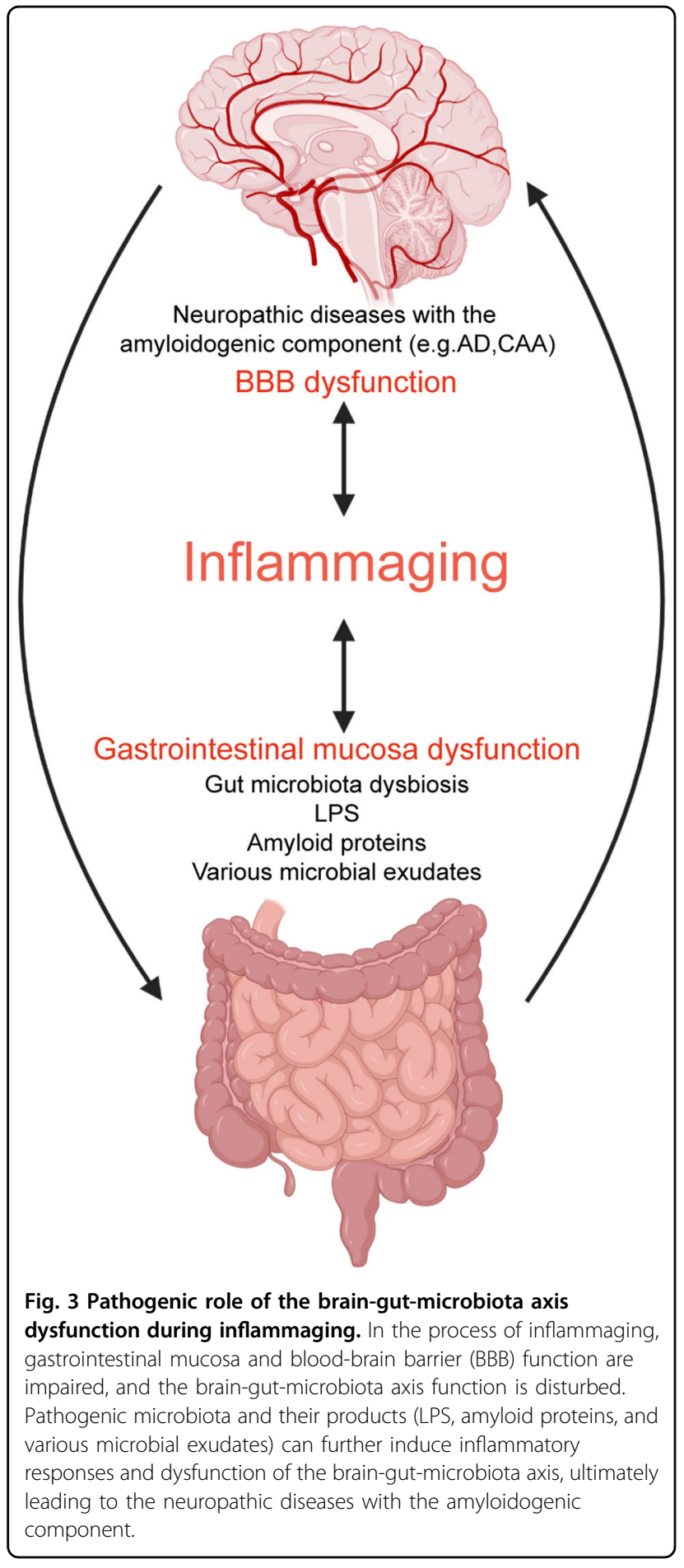

controlling vascular risk factors, antiplatelet therapy, intravenous thrombolysis, etc. ${ }^{1}$. In particular, antiplatelet and thrombolytic therapy reduces the risk of ischemic stroke but increases the risk of cerebral hemorrhage as well. Thus, it is necessary to balance the relationship between these two aspects to ensure the maximum benefit of treatment ${ }^{109}$.
With the deepening understanding of the pathogenesis of age-related CSVD, new therapeutic interventions have been proposed (Table 1). Recently, specific therapeutic targets for inflammaging have attracted increasing attention. Shuzhen $\mathrm{Z}$ et al. ${ }^{110}$ proposed that lipoproteinassociated phospholipase A2 (Lp-PLA2) and superoxide dismutase (SOD), were independently related to cognitive dysfunction and WMH lesion of CSVD, and could be considered as therapeutic targets to prevent age-related CSVD. Several anti-inflammatory drugs, such as fingolimod, natalizumab, dimethyl fumarate, and rituximab, have been applied to treat neuroinflammatory diseases such as multiple sclerosis ${ }^{54}$. Ongoing studies are evaluating the effectiveness of anti-inflammatory drugs for CSVD, but clinical evidence is still lacking ${ }^{109}$.

An important pathophysiological mechanism of CSVD, endothelium dysfunction and subsequent BBB leakage, provides an option for the targeted therapy of CSVD. In rat models of CSVD, ECs dysfunction was found to be the first change in the development of CSVD, and treatment with EC-stabilizing drugs (e.g., statins, ACEIs, cilostazol) could reverse these EC and oligodendroglial pathologies $^{111}$. In clinical trials, statins have previously been shown to reduce stroke rates in CSVD patients, but only in patients with elevated cholesterol ${ }^{112}$. Trials using ACEIs alone, excluding other types of antihypertensive agents, have shown a more active role in preventing WMH progression in CSVD $^{113}$. Cilostazol is currently undergoing early stage clinical trials for CSVD. In addition, pericyte is an under-studied cell type that wraps the ECs and is embedded in the basement membrane outside brain vessels including capillaries, post-capillary venules, and terminal arterioles ${ }^{114}$. Pericytes play a vital role in regulating various microvascular functions, such as angiogenesis, BBB preservation, capillary blood flow, and the migration of immune cells into the brain. Molecules mediating pericyte-EC interactions, such as plateletderived growth factor-BB (PDGF-BB), TGF- $\beta$, have been proposed as targets for the treatment of neurological disorders $^{115}$. In a cell culture model, using PDGF-BB or TGF- $\beta$ could better maintain BBB function under hypoxia conditions ${ }^{116}$. However, in CSVD, animal and clinical trials of relevant drugs have not yet been conducted.

Stem cell therapy has attracted increasing attention in recent years. Intravenous infusion of MSCs have been shown to inhibit the deterioration of BBB function and improves the functional outcome in a middle cerebral artery occlusion (MCAO) model ${ }^{117}$. In a CSVD model SHR (stroke-prone) with impaired cognitive, the infusion of MSCs was found to exert a therapeutic effect on CSVD. It could restore the $\mathrm{BBB}$ function via remodeling of microvasculature and reduce the $A \beta$ accumulation, by which inhibiting progressive brain atrophy and restoring cognitive dysfunction ${ }^{118}$. Therefore, the intravenous 
Table 1 New progress in therapeutic interventions of age-related CSVD.

\begin{tabular}{|c|c|c|c|}
\hline Targets & Mechanisms of action & Intervention Strategies & References \\
\hline Vascular/neuro-inflammation & $\begin{array}{l}\text {-Regulate the differentiation and migration of lymphocytes } \\
\text {-Impact the anti-oxidative stress cell machinery }\end{array}$ & $\begin{array}{l}\text {-Anti-inflammatory drugs } \\
\text {-Fingolimod } \cdot \text { Natalizumab } \\
\text {-Dimethyl fumarate } \\
\text {-Rituximab }\end{array}$ & $54,109,110$ \\
\hline Integrity of the BBB & $\begin{array}{l}\text {-Maintain the stability of endothelial function } \\
\text {-Promote interactions between pericytes and ECs }\end{array}$ & $\begin{array}{l}\text {-EC-stabilizing drugs } \\
\cdot \text { Statins } \\
\cdot \text { ACEls } \\
\cdot \text { Cilostazol }\end{array}$ & $111-116$ \\
\hline Stem cell & $\begin{array}{l}\text {-Remodel of microvasculature through MSCs } \\
\text {-Reprogram of pericytes to promote vasculogenesis }\end{array}$ & $\begin{array}{l}\text {-Intravenous infusion of MSCs } \\
\text {-Intracranial pericytes implantation }\end{array}$ & $117-122$ \\
\hline
\end{tabular}

administration of MSCs would be a new and reasonable therapeutic strategy for age-related CSVD. Moreover, a growing body of evidence indicates that pericytes are multipotential stem cells ${ }^{119}$. Recent studies have demonstrated that pericytes are capable to differentiate into neurons, microglia, and vasculature after brain injuries in ischemic disease and hypoxia conditions ${ }^{120,121}$. In amyloid model mice, pericyte implantation in the brain increased cerebral blood flow and reduced pathological deposition of $A \beta^{122}$. These findings suggest that transplantation of pericytes may be a promising approach to treat agerelated CSVD.

\section{Future directions}

Although considered to be independent lesions in the past, studies have suggested that DPA and CAA interact and overlap in pathological mechanisms, which can be regarded as the extreme outcomes of the age-related CSVD development ${ }^{2}$. Given the above-mentioned effects of inflammaging on systemic and vascular inflammation, it may help to explain the pathophysiological interactions between DPA and CAA.

Gut microbiota dysbiosis is an essential link between inflammaging and CSVD. However, most of the current studies focus on the relationship between gut microbiota and neurodegenerative diseases (e.g., Alzheimer's disease). Future research should focus on the co-relationship between gut microbiota of age-related CSVD patients and inflammaging.

At present, therapeutic interventions of age-related CSVD targeted for inflammaging, endothelium dysfunction, and subsequent BBB leakage, have been proposed, but most of them only enter the molecular and animal experimental stage. In the future, more clinical trials should be conducted to evaluate their safety and effectiveness.

\section{Conclusion}

Aged individuals commonly undergo a chronic sterile low-grade inflammation called inflammaging, in which a variety of cellular and biological mechanisms play a role. In the elderly, inflammaging predicts the risk of various age-related chronic non-infectious diseases, including age-related CSVD. There are two main types of agerelated CSVD, namely DPA and CAA. Regional analyses show that systemic and vascular inflammation have correlations with two distinct types of CSVD respectively. The close relationship between inflammaging and agerelated CSVD suggests the potential for inflammagingrelated therapy. However, few studies have focused on inflammaging-related therapies and their effects on agerelated CSVD. This gap is an important area of unmet medical need that deserves further translational research.

\section{Acknowledgements}

This study was financially supported by the National Natural Science Foundation of China (NSFC) (No.81671178 and 81971110), Guangzhou Science and Technology Program key projects (No.202007030010) and Guangzhou Science, Technology and Innovation Commission (Bureau of Science and Information Technology of Guangzhou Municipality) (No.201704020222) to Z.L.

\section{Author details}

'Department of Neurology, the Third Affiliated Hospital of Sun Yat-sen University, Guangzhou, Guangdong 510630, China. ${ }^{2}$ Mental and Neurological Diseases Research Center, the Third Affiliated Hospital of Sun Yat-sen University, Guangzhou, Guangdong 510630, China. ${ }^{3}$ Department of Endocrinology and Diabetes Center, the First Affiliated Hospital of Sun Yat-sen University, Guangzhou, Guangdong 510080, China

\section{Conflict of interest}

The authors declare that the research was conducted in the absence of any commercial or financial relationships that could be construed as a potential conflict of interest.

Publisher's note

Springer Nature remains neutral with regard to jurisdictional claims in published maps and institutional affiliations. 
Received: 16 June 2020 Revised: 10 October 2020 Accepted: 13 October 2020

Published online: 30 October 2020

\section{References}

1. Cannistraro, R. J. et al. CNS small vessel disease: a clinical review. Neurology 92, 1146-1156 (2019)

2. Schreiber, S. et al. Invited Review: the spectrum of age-related small vessel diseases: potential overlap and interactions of amyloid and nonamyloid vasculopathies. Neuropathol. Appl. Neurobiol. 46, 219-239 (2020).

3. Franceschi, C., Garagnani, P., Parini, P., Giuliani, C. \& Santoro, A. Inflammaging: a new immune-metabolic viewpoint for age-related diseases. Nat. Rev. Endocrinol. 14, 576-590 (2018).

4. Franceschi, $C$. et al. Inflamm-aging. An evolutionary perspective on immunosenescence. Ann. N. Y. Acad. Sci. 908, 244-254 (2000).

5. Vitale, G., Salvioli, S. \& Franceschi, C. Oxidative stress and the ageing endocrine system. Nat. Rev. Endocrinol. 9, 228-240 (2013).

6. Low, A., Mak, E., Rowe, J. B., Markus, H. S. \& O'Brien, J. T. Inflammation and cerebral small vessel disease: A systematic review. Ageing Res. Rev. 53, 100916 (2019).

7. Kennedy, B. K. et al. Geroscience: linking aging to chronic disease. Cell 159 709-713 (2014).

8. Gregor, M. F. \& Hotamisligil, G. S. Inflammatory mechanisms in obesity. Annu. Rev. Immunol. 29, 415-445 (2011).

9. Gay, N. J. \& Gangloff, M. Structure and function of toll receptors and their Ligands. Annu. Rev. Biochem. 76, 141-165 (2007).

10. Tieri, P. et al. Network, degeneracy and bow tie. Integrating paradigms and architectures to grasp the complexity of the immune system.Theor. Biol. Med. Model 7, 32 (2010).

11. Sanada, F. et al. Source of chronic inflammation in aging. Front. Cardiovasc. Med. 5, 12 (2018).

12. Franceschi, C. \& Campisi, J. Chronic inflammation (inflammaging) and its potential contribution to age-associated diseases. J. Gerontol. A Biol. Sci. Med. Sci. 69(Suppl 1), S4-S9 (2014).

13. Robbins, P. D. Extracellular vesicles and aging. Stem Cell Investig. 4, 98 (2017).

14. Franceschi, C., Garagnani, P., Vitale, G., Capri, M. \& Salvioli, S. Inflammaging and 'Garb-aging'. Trends Endocrinol. Metab. 28, 199-212 (2017).

15. Chazaud, B. \& Mouchiroud, G. Inflamm-aging: STAT3 signaling pushes muscle stem cells off balance. Cell Stem Cell 15, 401-402 (2014).

16. Salminen, A. et al. Activation of innate immunity system during aging: NF-kB signaling is the molecular culprit of inflamm-aging. Ageing Res. Rev. 7, 83-105 (2008).

17. Munir, H., Ward, L. S. C. \& McGettrick, H. M. Mesenchymal stem cells as endogenous regulators of inflammation. Adv. Exp. Med. Biol. 1060, 73-98 (2018).

18. Yu, K. R. \& Kang, K. S. Aging-related genes in mesenchymal stem cells: a minireview. Gerontology 59, 557-563 (2013).

19. Lee, B. C. \& Yu, K. R. Impact of mesenchymal stem cell senescence on inflammaging. BMB Rep. 53, 65-73 (2020).

20. Fulop, T. et al. Immunosenescence and inflamm-aging as two sides of the same coin: friends or foes? Front Immunol. 8, 1960 (2017).

21. Pawelec, G. Age and immunity: what is "immunosenescence"? Exp. Gerontol. 105, 4-9 (2018).

22. Simon, A. K., Hollander, G. A. \& McMichael, A. Evolution of the immune system in humans from infancy to old age. Proc. Biol. Sci. 282, 20143085 (2015).

23. Solana, R. et al. Innate immunosenescence: effect of aging on cells and receptors of the innate immune system in humans. Semin. Immunol. 24, 331-341 (2012).

24. Pinti, M. et al. Aging of the immune system: focus on inflammation and vaccination. Eur. J. Immunol. 46, 2286-2301 (2016).

25. Weng, N.P. Aging of the immune system: how much can the adaptive immune system adapt? Immunity 24, 495-499 (2006).

26. Salminen, A., Kaarniranta, K. \& Kauppinen, A. Immunosenescence: the potential role of myeloid-derived suppressor cells (MDSC) in age-related immune deficiency. Cell Mol. Life Sci. 76, 1901-1918 (2019).

27. Miller, J. P. \& Cancro, M. P. B cells and aging: balancing the homeostatic equation. Exp. Gerontol. 42, 396-399 (2007)

28. Frasca, D. Senescent B cells in aging and age-related diseases: their role in the regulation of antibody responses. Exp. Gerontol. 107, 55-58 (2018).
29. Soto-Heredero, G., Baixauli, F. \& Mittelbrunn, M. Interorganelle communication between mitochondria and the endolysosomal system. Front. Cell Dev. Biol. 5, 95 (2017).

30. Desdín-Micó, G., Soto-Heredero, G. \& Mittelbrunn, M. Mitochondrial activity in T cells. Mitochondrion 41, 51-57 (2018).

31. Harper, J. W., Ordureau, A. \& Heo, J. M. Building and decoding ubiquitin chains for mitophagy. Nat. Rev. Mol. Cell Biol. 19, 93-108 (2018).

32. Green, D. R., Galluzzi, L. \& Kroemer, G. Mitochondria and the autophagyinflammation-cell death axis in organismal aging. Science 333, 1109-1112 (2011).

33. Nakahira, K. et al. Autophagy proteins regulate innate immune responses by inhibiting the release of mitochondrial DNA mediated by the NALP3 inflammasome. Nat. Immunol. 12, 222-230 (2011).

34. Oka, T. et al. Mitochondrial DNA that escapes from autophagy causes inflammation and heart failure. Nature 485, 251-255 (2012).

35. Feske, S. Calcium signalling in lymphocyte activation and disease. Nat. Rev. Immunol. 7, 690-702 (2007).

36. Nathan, C. \& Cunningham-Bussel, A. Beyond oxidative stress: an immunologist's guide to reactive oxygen species. Nat. Rev. Immunol. 13, 349-361 (2013).

37. Martinvalet, D. The role of the mitochondria and the endoplasmic reticulum contact sites in the development of the immune responses. Cell Death Dis. $\mathbf{9}$, 336 (2018).

38. De la Fuente, M. \& Miquel, J. An update of the oxidation-inflammation theory of aging: the involvement of the immune system in oxi-inflamm-aging. Curr. Pharm. Des. 15, 3003-3026 (2009).

39. Uysal, K. T., Wiesbrock, S. M., Marino, M. W. \& Hotamisligil, G. S. Protection from obesity-induced insulin resistance in mice lacking TNF-alpha function. Nature 389, 610-614 (1997)

40. Hotamisligil, G. S., Shargill, N. S. \& Spiegelman, B. M. Adipose expression of tumor necrosis factor-alpha: direct role in obesity-linked insulin resistance. Science 259, 87-91 (1993).

41. Reilly, S. M. \& Saltiel, A. R. Adapting to obesity with adipose tissue inflammation. Nat. Rev. Endocrinol. 13, 633-643 (2017).

42. Halter, J. B. et al. Diabetes and cardiovascular disease in older adults: current status and future directions. Diabetes 63, 2578-2589 (2014).

43. van Oostrom, A. J. H. H. M. et al. Activation of leukocytes by postprandial lipemia in healthy volunteers. Atherosclerosis 177, 175-182 (2004).

44. Sampson, M. J., Davies, I. R., Brown, J. C., Ivory, K. \& Hughes, D. A. Monocyte and neutrophil adhesion molecule expression during acute hyperglycemia and after antioxidant treatment in type 2 diabetes and control patients. Arterioscler. Thromb. Vasc. Biol. 22, 1187-1193 (2002).

45. Alipour, A. et al. Leukocyte activation by triglyceride-rich lipoproteins. Arterioscler. Thromb. Vasc. Biol. 28, 792-797 (2008).

46. Collino, S. et al. Metabolic signatures of extreme longevity in northern Italian centenarians reveal a complex remodeling of lipids, amino acids, and gut microbiota metabolism. PLOS ONE 8, e56564 (2013).

47. Moeller, A. H. et al. Cospeciation of gut microbiota with hominids. Science 353, 380-382 (2016)

48. Groussin, M. et al. Unraveling the processes shaping mammalian gut microbiomes over evolutionary time. Nat. Commun. 8, 14319 (2017).

49. Santoro, A. et al. Gut microbiota changes in the extreme decades of human life: a focus on centenarians. Cell Mol. Life Sci. 75, 129-148 (2018).

50. Biagi, E. et al. Gut microbiota and extreme longevity. Curr. Biol. 26, 1480-1485 (2016).

51. Biagi, E. et al. Through ageing, and beyond: gut microbiota and inflammatory status in seniors and centenarians. PLOS ONE 5, e10667 (2010).

52. Thevaranjan, N. et al. Age-associated microbial dysbiosis promotes intestinal permeability, systemic inflammation, and macrophage dysfunction. Cell Host. Microbe. 21, 455-466 (2017).

53. Fransen, F. et al. Aged gut microbiota contributes to systemical inflammaging after transfer to germ-free mice. Front. Immunol. 8, 1385 (2017).

54. Fu, Y. \& Yan, Y. Emerging role of immunity in cerebral small vessel disease. Front. Immunol. 9, 67 (2018).

55. Calder, P. C. et al. Health relevance of the modification of low grade inflammation in ageing (inflammageing) and the role of nutritionAgeing Res. Rev. 40, 95-119 (2017).

56. Staals, J. et al. Total MRI load of cerebral small vessel disease and cognitive ability in older people. Neurobiol. Aging 36, 2806-2811 (2015).

57. Pantoni, L. Cerebral small vessel disease: from pathogenesis and clinical characteristics to therapeutic challenges. Lancet Neurol. 9, 689-701 (2010). 
58. Charidimou, A. et al. Enlarged perivascular spaces as a marker of underlying arteriopathy in intracerebral haemorrhage: a multicentre MRI cohort study. J. Neurol. Neurosurg. Psychiatry 84, 624-629 (2013).

59. Greenberg, S. M. et al. Cerebral microbleeds: a guide to detection and interpretation. Lancet Neurol. 8, 165-174 (2009).

60. Kario, K. et al. Silent cerebral infarcts in basal ganglia are advanced in congenital protein C-deficient heterozygotes with hypertension. Am. J. Hypertens. 14, 818-822 (2001).

61. Jandke, S. et al. The association between hypertensive arteriopathy and cerebral amyloid angiopathy in spontaneously hypertensive stroke-prone rats. Brain Pathol. 28, 844-859 (2018).

62. Liberale, L. et al. Post-ischaemic administration of the murine Canakinumabsurrogate antibody improves outcome in experimental stroke. Eur. Heart $\mathrm{J}$. 39, 3511-3517 (2018)

63. Bonaventura, A. et al. The pathophysiological role of neutrophil extracellular traps in inflammatory diseases.Thromb. Haemost. 118, 6-27 (2018).

64. Kaiser, D. et al. Spontaneous white matter damage, cognitive decline and neuroinflammation in middle-aged hypertensive rats: an animal model of early-stage cerebral small vessel disease. Acta Neuropathol. Commun. 2, 169 (2014).

65. Senchenkova, E. Y., Russell, J., Kurmaeva, E., Ostanin, D. \& Granger, D. N. Role of $\mathrm{T}$ lymphocytes in angiotensin I-mediated microvascular thrombosis. Hypertension 58, 959-965 (2011)

66. Kimura, A. et al. Anti-endothelial cell antibodies in patients with cerebral small vessel disease. Curr. Neurovasc Res. 9, 296-301 (2012).

67. Liberale, L., Dallegri, F., Montecucco, F. \& Carbone, F. Pathophysiological relevance of macrophage subsets in atherogenesis.Thromb. Haemost. 117 7-18 (2017).

68. Virdis, A., Neves, M. F., Amiri, F., Touyz, R. M. \& Schiffrin, E. L. Role of NAD(P)H oxidase on vascular alterations in angiotensin II-infused mice. J. Hypertens. 22 535-542 (2004).

69. Broz, P. \& Dixit, V. M. Inflammasomes: mechanism of assembly, regulation and signalling. Nat. Rev. Immunol. 16, 407-420 (2016).

70. Vanaja, S. K., Rathinam, V. A. K. \& Fitzgerald, K. A. Mechanisms of inflammasome activation: recent advances and novel insights. Trends Cell Biol. 25 308-315 (2015).

71. Swanson, K. V., Deng, M. \& Ting, J. P. Y. The NLRP3 inflammasome: molecular activation and regulation to therapeutics. Nat. Rev. Immunol. 19, 477-489 (2019).

72. Tomiyama, $\mathrm{H}$. et al. The contribution of inflammation to the development of hypertension mediated by increased arterial stiffness. Jm. Heart Assoc. 6 , e005729 (2017).

73. Liu, D., Zeng, X., Li, X., Mehta, J. L. \& Wang, X. Role of NLRP3 inflammasome in the pathogenesis of cardiovascular diseases. Basic Res. Cardiol. 113, 5 (2018).

74. Libby, P. Interleukin-1 beta as a target for atherosclerosis therapy: biological basis of CANTOS and beyond. J. Am. Coll. Cardiol. 70, 2278-2289 (2017).

75. Libby, P. \& Rocha, V. Z. All roads lead to IL-6: A central hub of cardiometabolic signaling. Int. J. Cardiol. 259, 213-215 (2018).

76. Matthews, C. et al. Vascular smooth muscle cells undergo telomere-based senescence in human atherosclerosis: effects of telomerase and oxidative stress. Circ. Res. 99, 156-164 (2006).

77. Minamino, T. et al. Endothelial cell senescence in human atherosclerosis: role of telomere in endothelial dysfunction. Circulation 105 1541-1544 (2002)

78. Childs, B. G., Durik, M., Baker, D. J. \& van Deursen, J. M. Cellular senescence in aging and age-related disease: from mechanisms to therapy. Nat. Med. 21, 1424-1435 (2015).

79. Wang, M., Kim, S. H., Monticone, R. E. \& Lakatta, E. G. Matrix metalloproteinases promote arterial remodeling in aging, hypertension, and atherosclerosis. Hypertension 65, 698-703 (2015).

80. Libby, P. Mechanisms of acute coronary syndromes and their implications for therapy. N. Engl. J. Med. 368, 2004-2013 (2013).

81. Wang, Y. I. et al. Triglyceride-rich lipoprotein modulates endothelial vascular cell adhesion molecule (VCAM)-1 expression via differential regulation of endoplasmic reticulum stress. PLOS ONE 8, e78322 (2013).

82. Higgins, L. J. \& Rutledge, J. C. Inflammation associated with the postprandial lipolysis of triglyceride-rich lipoproteins by lipoprotein lipase. Curr. Atheroscler. Rep. 11, 199-205 (2009).

83. Stout, M. B., Justice, J. N., Nicklas, B. J. \& Kirkland, J. L. Physiological aging: links among adipose tissue dysfunction, diabetes, and frailty.Physiology 32, 9-19 (2017)
84. Weisberg, S. P. et al. Obesity is associated with macrophage accumulation in adipose tissue. J. Clin. Investig. 112, 1796-1808 (2003).

85. Libby, P. et al. Inflammation, immunity, and infection in atherothrombosis: JACC review topic of the week. J. Am. Coll. Cardiol. 72, 2071-2081 (2018).

86. Lindskog Jonsson, A. et al. Bacterial profile in human atherosclerotic plaques. Atherosclerosis 263, 177-183 (2017).

87. Attems, J., Jellinger, K., Thal, D. R. \& Van Nostrand, W. Review: sporadic cerebral amyloid angiopathy. Neuropathol. Appl. Neurobiol. 37, 75-93 (2011)

88. Tarasoff-Conway, J. M. et al. Clearance systems in the brain-implications for Alzheimer disease. Nat. Rev. Neurol. 11, 457-470 (2015).

89. Nedergaard, M. Neuroscience Garbage truck of the brain. Science $\mathbf{3 4 0}$ 1529-1530 (2013).

90. Tejera, D. \& Heneka, M. T. Microglia in Alzheimer's disease: the good, the bad and the ugly. Curr. Alzheimer Res. 13, 370-380 (2016).

91. Wyss-Coray, T. \& Rogers, J. Inflammation in Alzheimer disease-a brief review of the basic science and clinical literature. Cold Spring Harb. Perspect. Med. 2, a006346 (2012).

92. Biber, K., Neumann, H., Inoue, K. \& Boddeke, H. W. G. M. Neuronal 'On' and 'Off' signals control microglia. Trends Neurosci. 30, 596-602 (2007).

93. Lu, A. et al. Unified polymerization mechanism for the assembly of ASCdependent inflammasomes. Cell 156, 1193-1206 (2014).

94. Walsh, J. G., Muruve, D. A. \& Power, C. Inflammasomes in the CNS. Nat. Rev. Neurosci. 15, 84-97 (2014).

95. Tejera, D. et al. Systemic inflammation impairs microglial $A B$ clearance through NLRP3 inflammasome. EMBO J. 38, e101064 (2019).

96. Chen, $Y$. et al. Instigation of endothelial Nlrp3 inflammasome by adipokine visfatin promotes inter-endothelial junction disruption: role of HMGB1. J. Cell Mol. Med. 19, 2715-2727 (2015)

97. Paneni, F., Diaz Cañestro, C., Libby, P., Lüscher, T. F. \& Camici, G. G. The aging cardiovascular system: understanding it at the cellular and clinical levels. J. Am. Coll. Cardiol. 69, 1952-1967 (2017).

98. Ferretti, M. T. et al. T-cell brain infiltration and immature antigen-presenting cells in transgenic models of Alzheimer's disease-like cerebral amyloidosis. Brain Behav. Immun. 54, 211-225 (2016).

99. Dinan, T. G. \& Cryan, J. F. Gut instincts: microbiota as a key regulator of brain development, ageing and neurodegeneration. J. Physiol. 595, 489-503 (2017).

100. Quigley, E. M. M. Microbiota-brain-gut axis and neurodegenerative diseases. Curr. Neurol. Neurosci. Rep. 17, 94 (2017).

101. Köhler, C. A. et al. The gut-brain axis, including the microbiome, leaky gut and bacterial translocation: mechanisms and pathophysiological role in Alzheimer's disease. Curr. Pharm. Des. 22, 6152-6166 (2016).

102. Yano, J. M. et al. Indigenous bacteria from the gut microbiota regulate host serotonin biosynthesis. Cell 161, 264-276 (2015).

103. Taylor, J. D. \& Matthews, S. J. New insight into the molecular control of bacterial functional amyloids. Front Cell Infect. Microbiol. 5, 33 (2015).

104. Hill, J. M. \& Lukiw, W. J. Microbial-generated amyloids and Alzheimer's disease (AD). Front. Aging Neurosci. 7, 9 (2015).

105. Zhao, Y. \& Lukiw, W. J. Microbiome-generated amyloid and potential impact on amyloidogenesis in Alzheimer's disease (AD). J. Nat. Sci. 1, e138 (2015).

106. Lukiw, W. J. Lipopolysaccharide and inflammatory signaling in Alzheimer's disease. Front. Microbiol. 7, 1544 (2016).

107. Zhao, Y., Dua, P. \& Lukiw, W. J. Microbial sources of amyloid and relevance to amyloidogenesis and Alzheimer's Disease (AD). J. Alzheimers Dis. Parkinsonism 5, 177 (2015).

108. Gąsiorowski, K., Brokos, B., Echeverria, V., Barreto, G. E. \& Leszek, J. RAGE-TLR crosstalk sustains chronic inflammation in neurodegeneration. Mol. Neurobiol. 55, 1463-1476 (2018).

109. Bath, P. M. \& Wardlaw, J. M. Pharmacological treatment and prevention of cerebral small vessel disease: a review of potential interventions. Int. J. Stroke 10, 469-478 (2015)

110. Zhu, S. et al. Plasma lipoprotein-associated phospholipase A2 and superoxide dismutase are independent predicators of cognitive impairment in cerebra small vessel disease patients: diagnosis and assessment. Aging Dis. 10, 834-846 (2019).

111. Rajani, R. M. et al. Reversal of endothelial dysfunction reduces white matter vulnerability in cerebral small vessel disease in rats. Sci. Transl. Med. $\mathbf{1 0}$ eaam9507 (2018)

112. $\mathrm{Fu}, \mathrm{J} . \mathrm{H}$. et al. Effects of statins on progression of subclinical brain infarct. Cerebrovasc. Dis. 30, 51-56 (2010). 
113. Dufouil, C. et al. Effects of blood pressure lowering on cerebral white matter hyperintensities in patients with stroke: the PROGRESS (Perindopril Protection Against Recurrent Stroke Study) Magnetic Resonance Imaging Substudy. Circulation 112, 1644-1650 (2005).

114. Armulik, A., Genové, G. \& Betsholtz, C. Pericytes: developmental, physiological, and pathological perspectives, problems, and promises. Dev. Cell 21, 193-215 (2011)

115. Cheng, J. et al. Targeting pericytes for therapeutic approaches to neurological disorders. Acta neuropathologica 136, 507-523 (2018).

116. Shen, J. et al. PDGFR- $\beta$ restores blood-brain barrier functions in a mouse mode of focal cerebral ischemia. J. Cereb. Blood Flow. Metab. 39, 1501-1515 (2019).

117. Nakazaki, M. et al. Intravenous infusion of mesenchymal stem cells inhibits intracranial hemorrhage after recombinant tissue plasminogen activator therapy for transient middle cerebral artery occlusion in rats. J. Neurosurg. 127, 917-926 (2017).
118. Nakazaki, M. et al. Intravenous infusion of mesenchymal stem cells improves impaired cognitive function in a cerebral small vessel disease model. Neuroscience 408, 361-377 (2019).

119. Dore-Duffy, P. Pericytes: pluripotent cells of the blood brain barrier. Curr. Pharm. Des. 14, 1581-1593 (2008).

120. Nakagomi, T. et al. Brain vascular pericytes following ischemia have multipotential stem cell activity to differentiate into neural and vascular lineage cells. Stem Cells 33, 1962-1974 (2015).

121. Özen, I. et al. Brain pericytes acquire a microglial phenotype after stroke. Acta Neuropathologica 128, 381-396 (2014).

122. Tachibana, M., Yamazaki, Y., Liu, C.-C., Bu, G. \& Kanekiyo, T. Pericyte implantation in the brain enhances cerebral blood flow and reduces amyloid- $\beta$ pathology in amyloid model mice. Exp. Neurol. 300, 13-21 (2018). 